\title{
Entre Ilustración y Romanticismo: Fichte y el legado de Rousseau
}

Frederick Neuhouser

Traductor. Emiliano Acosta

(2) OpenEdition

Journals

Edición electrónica

URL: http://journals.openedition.org/ref/448

DOI: $10.4000 /$ ref.448

ISSN: 2258-014X

Editor

EuroPhilosophie Editions

\section{Referencia electrónica}

Frederick Neuhouser, «Entre llustración y Romanticismo: Fichte y el legado de Rousseau », Revista de Estud(i)os sobre Fichte [En línea], 7 | 2013, Publicado el 30 diciembre 2013, consultado el 08

septiembre 2020. URL : http://journals.openedition.org/ref/448 ; DOI : https://doi.org/10.4000/ref.448

Este documento fue generado automáticamente el 8 septiembre 2020.

(c) EuroPhilosophie 


\title{
Entre Ilustración y Romanticismo: Fichte y el legado de Rousseau
}

\author{
Frederick Neuhouser
}

Tradución : Emiliano Acosta

1 El presente artículo tratará aquello que podría ser considerado como la influencia romántica en el pensamiento de Fichte. Describir de este modo mi artículo puede de algún modo desorientar al lector dado que la figura cuya influencia en Fichte pretendo discutir, a saber, Jean-Jacques Rousseau, puede sólo en un sentido acotado ser visto como un romántico. Pues, como en el caso de Fichte, Rousseau también está atrapado entre los impulsos del romanticismo y de la Ilustración. Está fuera de toda discusión que Rousseau ejerció una gran influencia en Fichte, especialmente en las fases tempranas de su carrera. Es por esto que no debería sorprender que el pensamiento de Fichte, el cual ciertamente pertenece a la tradición de la Ilustración, refleje algunas de las actitudes ambivalentes de Rousseau con respecto a las ideas del romanticismo.

2 Hay muchos lugares en el pensamiento político de Fichte en los que la influencia de Rousseau puede ser detectada, por ejemplo, en su concepción del contrato social, su sensibilidad ante los males que acarrea la inequidad económica y su insistencia en que instituciones políticas buenas dependen de que existan entre los ciudadanos lazos de solidaridad y no solamente relaciones recíprocas entre intereses privados. Pero tal vez la idea más importante que Fichte heredó de Rousseau sea una cierta concepción de aquello en lo que consiste la tarea general de la filosofía -una concepción que recibe su forma de aquello que debería ser considerado el ideal romántico de la reconciliación entre naturaleza y cultura humana, o, dicho con mayor precisión, reconciliación entre nuestros fines naturales y nuestros fines espirituales. ${ }^{1}$

3 En el pensamiento de Rousseau este proyecto es llevado a cabo en sus tres textos filosóficos centrales: el Discurso sobre el Origen de la Inequidad, Emilio y El contrato social. En el Discurso sobre el Origen de la Inequidad Rousseau describe las distintas maneras en las que los fines naturales y espirituales pueden entrar en conflicto a medida que la cultura se desarrolla, mientras que en el Emilio y en El contrato social Rousseau demuestra cómo la cultura en sí misma -educación nacional de un lado, e instituciones políticas del 
otro- posee por lo menos el potencial para resolver este conflicto, el cual es por cierto imposible de ignorar.

Permítanme comenzar recordándoles cómo Kant, otro discípulo de Rousseau, describe en su escrito "Inicio conjetural de la historia humana" aquello que llamo el proyecto romántico de Rousseau (el cual es al mismo tiempo el proyecto de la Ilustración):

[En sus Discursos Rousseau] pone en evidencia el conflicto inevitable entre cultura y naturaleza en el género humano considerado como una especie natural en la cual cada individuo debe consumar su destinación toda. Pero en su Emilio y en su Contrato social [...] intenta solucionar un problema aún más difícil, a saber: de qué manera tiene que progresar la cultura para así desarrollar las capacidades de la humanidad en concordancia con la destinación de la misma en cuanto especie moral de tal modo que la última no esté en conflicto con la destinación natural de la humanidad en cuanto especie natural." 2

El mejor modo de describir el "problema aún más difícil" al que Kant quiere que pongamos atención es presentarlo como un conflicto entre los fines que poseemos por el solo hecho de que somos seres naturales -supervivencia y felicidad, pero también el pleno desarrollo de nuestras capacidades naturales- $\mathrm{y}$ aquellos fines que poseemos por el solo hecho de que somos seres espirituales: racionalidad, virtud moral y, lo más importante, libertad. La cultura es parte en este conflicto porque los desarrollos a los que nos sometemos al atender a nuestras necesidades naturales -cultura, en otras palabras - nos van cambiando de tal modo que virtud y libertad se vuelven cada vez más difíciles de alcanzar. El Segundo Discurso de Rousseau describe la cantidad de obstáculos que los seres humanos civilizados afrontan al realizar al mismo tiempo fines naturales y espirituales -aquí debemos recordar que el amour-propre, esto es, el impulso de reconocimiento social, juega un rol mayor en ese asunto- pero sus sucesores parecen asentir en que él tuvo mayor éxito en comprender los problemas complejos que afrontamos como resultado del desarrollo cultural que en resolverlos. ${ }^{3}$ No significa ir demasiado lejos decir que Fichte y Kant retomaron el problema de Rousseau y lo convirtieron en la tarea que definió sus filosofías.

6 Hay muchos aspectos del pensamiento de Fichte que podrían ser señalados como evidencia a favor de mi tesis de que el deseo de resolver el problema que Rousseau plantea en su Segundo Discurso es la fuerza motivadora detrás del pensamiento de Fichte tomado en conjunto. Consideren, por ejemplo, la afirmación que hace en BG: "cultura [...] es el medio último y supremo para alcanzar el fin final del ser humano, concordancia [Übereinstimmung] completa con uno mismo" ${ }^{4}$, un fin que Fichte entiende en términos de armonía entre cuatro elementos: sentimiento, el desarrollo de nuestros talentos naturales, virtud moral y libertad. En una variación atenuada de las conclusiones de Rousseau Fichte continua argumentando -en todas sus obras principales- que incluso tal armonía nunca podrá ser alcanzada completamente por ser humano alguno. Nuestra naturaleza es tal, sin embargo, que es posible para nosotros hacer progresos substanciales hacia ese fin.

7 Aun cuando ambos pensadores concuerden en que la naturaleza permite tal progreso, Fichte es en lo que concierne a la probabilidad de que tal progreso llegue a suceder mucho más optimista que Rousseau. Como Kant, y a diferencia de Rousseau, Fichte ha hecho propia cierta versión de una actitud típica de la Ilustración para con la historia, según la cual la naturaleza misma, especialmente la naturaleza humana, contiene dentro de sí semillas o tendencias -ciertos impulsos psicológicos, por ejemplo ${ }^{5}-$ que 
hacen, si no estrictamente necesario, al menos muy posible, el progreso hacia la consecución de nuestros fines espirituales. ${ }^{6}$

Yo podría argumentar que la actitud más optimista de Fichte hacia la historia humana se debe a cierta falencia para comprender la gran complejidad del problema tal como Rousseau lo presenta en su Segundo Discurso. ${ }^{7}$ Digámoslo de un modo más preciso: Fichte no alcanza a ver la cantidad de obstáculos inevitables que nuestro amour-propre nuestra pasión por ser estimados o valorados por nuestros semejantes- impone a nuestra capacidad para ser felices y libres, una vez que la pasión ha sido despertada y moldeada por el desarrollo cultural. ${ }^{8} \mathrm{Al}$ mismo tiempo -este es el argumento que deseo llevar adelante hoy- el optimismo de Fichte con respecto a la historia lo lleva a descubrir en la naturaleza recursos importantes que le ayudan a, y pueden permitirnos a nosotros, temperar la visión excesivamente pesimista de la condición humana. El aspecto del pensamiento de Fichte que me propongo investigar aquí es el uso que hace de la idea de Anerkennung o reconocimiento -el fin que nuestro amour-propre nos lleva a buscar- en su concepción positiva de cómo los seres humanos pueden realizar virtud, libertad y racionalidad, todo a la vez. Busco, en otras palabras, examinar cómo Fichte se apropia de la teoría, ciertamente compleja, de Rousseau acerca del amour-propre y del reconocimiento humano. Mi tesis es que aun cuando Fichte subestima los peligros que acechan al bienestar humano, los cuales provienen de nuestro impulso a ser reconocidos por nuestros semejantes, Fichte puede ser valorado ciertamente por haber descubierto en esa misma necesidad humana un potencial para dar cuenta de y evitar esos peligros de los cuales Rousseau no tuvo una noción lo suficientemente clara. ${ }^{9}$

Quiero comenzar con una recapitulación de la comprensión Rousseauniana del conflicto entre nuestros fines espirituales y naturales. Si bien Rousseau, al igual que Fichte, está preocupado con los fines de virtud y racionalidad, voy a limitarme aquí a examinar el modo en que, bajo las condiciones de civilización, la naturaleza, especialmente el hecho básico de la carencia original que caracteriza a todo ser humano, entra en conflicto con la libertad. El punto de partida de Rousseau es el carácter amenazante de la dependencia humana para la libertad. Dependencia debe entenderse aquí como lo opuesto a autosuficiencia, es decir, como la situación en la que las personas se ven obligadas a apoyarse en la cooperación de los otros para satisfacer sus propias necesidades. Rousseau expresa este aspecto de su visión cuando dice:

"puesto que los lazos de servidumbre están formados solamente por la mutua dependencia de los hombres y las necesidades recíprocas que los unen, es imposible hacer a un hombre esclavo [asservir] sin primero haberlo colocado en la situación de ser incapaz de hacer algo sin los otros." 10

El pensamiento detrás de esta afirmación es sencillo: una de las ideas centrales del Segundo Discurso es que la dependencia trae siempre consigo el peligro de que individuos dependientes tengan que comprometer su libertad para satisfacer así las necesidades que los obligan a cooperar con sus semejantes. Cualquier tipo de dependencia significa una amenaza para ser libre, dado que abre la posibilidad de que para obtener lo que necesito, tenga que amoldar mis acciones a las voluntades de aquellos con cuya cooperación cuento para satisfacer mis necesidades. Y esto -obedecer la voluntad de los otros- es la esencia de la dominación o pérdida de la libertad. Cuando uno se ve habitualmente confrontado con la disyuntiva entre tomar lo que necesito o 
seguir mi propia voluntad, no debería sorprender si finalmente la decisión de satisfacer mis necesidades se impone sobre la opción de seguir en libertad. Un ejemplo de esto en el Segundo Discurso es el rol que Rousseau da a la división del trabajo -en especial el desarrollo de la industria metalúrgica y la agricultura en cuanto ramas independientes de la producción- para explicar la ubicuidad de la dominación en el Estado civilizado. Es en primer lugar porque la división del trabajo aumenta la dependencia material entre los individuos y porque, en segundo lugar, pone en peligro sus libertades individuales, por lo que Rousseau dice que "hierro y trigo [...] civilizaron al hombre pero arruinaron al género humano."11

11 El segundo paso en el argumento de Rousseau concerniente al conflicto entre las necesidades y la libertad humanas consiste en que la amenaza que la dependencia representa para la libertad es exacerbada en gran medida cuando otro aspecto de la mayor parte de las sociedades humanas es introducido en la descripción, a saber: la desigualdad social. Aquí también la idea es sencilla: es mucho menos posible que la dependencia se traduzca en un daño para la libertad, si seres en interdependencia se encuentran unos con otros en pie de igualdad que si, desde el comienzo, una de las partes tiene ventaja en lo que respecta a riqueza, poder o prestigio. La desigualdad económica nos brinda el más claro ejemplo sobre este punto, si bien no es la única forma de desigualdad que Rousseau considera peligrosa. Adam Smith se limita a repetir el punto de Rousseau sobre los peligros que la inequidad representa para la libertad cuando él argumenta que ya que los trabajadores necesitan comer más urgentemente que lo que sus empleadores necesitan para hacer ganancias, éstos acaban casi siempre por declinar sus reclamos sobre las condiciones laborales en favor de las voluntades de sus empleadores. Por esto, los trabajadores ocupan siempre una posición en desventaja dentro de la relación de dependencia. ${ }^{12}$ Dicho con otras palabras: la dependencia se vuelve más peligrosa cuando viene unida a la inequidad social, puesto que esta mixtura tóxica amenaza la capacidad de algunos -los menos aventajados- para seguir lo que dictan sus voluntades.

12 Finalmente el impulso de reconocimiento entra en esta descripción porque el amourpropre trabaja en incrementar tanto la dependencia como la inequidad social. El amourpropre es una fuente substancial de necesidades para los humanos, dado que nos impulsa constantemente a esforzarnos por conseguir estima de nuestros semejantes, y la presión que ejerce sobre nosotros para que actuemos de ese modo sólo disminuye cuando tenemos suerte suficiente como para encontrar las formas y la cantidad de reconocimiento que creemos que merecemos. Además estas necesidades multiplican la dependencia humana porque el reconocimiento, a diferencia del alimento o del sueño, es un bien que sólo los otros pueden proveernos. Puesto que lo que el amour-propre busca es la buena opinión de los demás, obtenerla depende esencialmente de que los otros decidan entregárnosla libremente.

13 Existe, entonces, una conexión necesaria y directa entre amour-propre y dependencia. Es imposible para el impulso de reconocimiento ser satisfecho sin la participación de los otros. En tanto y en cuanto el amour-propre está presente en el mundo -esto es, en tanto y en cuanto haya seres humanos en el mundo- la dependencia será una característica permanente y dominante del mismo. ${ }^{13} \mathrm{El}$ amour-propre, ciertamente, es un fenómeno cultural antes que puramente natural, y el rol que juega en explicar la dependencia humana señala un importante punto para Rousseau: el conflicto entre nuestro depender de los otros y nuestra aptitud para ser libres no es en primer lugar un 
conflicto entre naturaleza y libertad, sino entre dependencia generada por medio de la cultura y libertad. Dicho con otras palabras: nuestras necesidades representan un problema para nuestra libertad, sólo una vez que la cultura ha transformado y multiplicado lo que necesitamos de los demás.

Al mismo tiempo que hace aumentar nuestra dependencia, el amour-propre es la fuente principal de la inequidad, la cual convierte la dependencia tan peligrosa para nuestra libertad. Esta es la afirmación fundamental del Segundo Discurso. El impulso de reconocimiento es la principal fuente de inequidad porque, a diferencia de las necesidades de alimento y sueño, nos provee de un incentivo para ir en búsqueda de inequidades por los propios bienes que ellas ofrecen. Esto se debe a que el amour-propre adopta fácilmente la forma de un deseo de alcanzar una posición superior con respecto a los demás. Una vez que adoptamos la actitud de mostrar cierto aprecio por los demás sólo a cambio de ser reconocidos como mejores que los otros, está todo listo para que se inicie un juego incesante de rivalidad en la que los individuos buscan constantemente nuevos modos de probar su superioridad. No debe sorprender, entonces, que el ámbito principal en que se da una competencia incesante sea el de la propiedad privada. El impulso de adquirir cada vez más grandes cantidades de prosperidad -y de allí el grado asombroso de inequidad económica que vemos en las sociedades en las que vivimos- es una consecuencia, no de nuestra necesidad de sobrevivir, sino de la necesidad mucho más inquieta de vencer a los otros para lograr formas inequitativas de estima social que nuestro amour-propre nos impulsa a buscar.

En suma, entonces, la carencia de los seres humanos civilizados -nuestra necesidad de sobrevivir así como también nuestra necesidad de ser reconocidos- se halla en conflicto básico con nuestra aptitud para ser libres (así como también para ser seres moral e intelectualmente virtuosos). La necesidad de preservarnos a nosotros mismos biológicamente nos exige que cooperemos con otros y que desarrollemos nuestras capacidades naturales a través de dicha cooperación. Pero ambos factores, al despertar el amour-propre y extenderlo a cada nuevo ámbito de la vida, aumentan nuestra dependencia y engendran nuevas formas de inequidad producto del interactuar humano. Cada una de estas formas lleva a una situación en la que se vuelve casi imposible seguir nuestra propia voluntad mientras sigamos satisfaciendo nuestras necesidades en vez de obedecer la voluntad de los otros.

Ya he sugerido que cuando Fichte intenta reconciliar naturaleza y cultura -cuando intenta encontrar una armonía posible entre los fines de felicidad, desarrollo humano, libertad y moralidad- subestima los obstáculos que, tal como Rousseau supo mostrar, el amour-propre impone para efectuar una reconciliación semejante. Puede ser, sin embargo, que esta falencia también le haya dado a Fichte la necesaria libertad como para descubrir en el impulso de reconocimiento un rol más positivo del que Rousseau parece haber sido consciente. ${ }^{14}$ En lo que sigue, consideraré las maneras, por cierto imprevistas desde una mirada Rousseauniana, en las que Fichte se apropia de la doctrina de Rousseau acerca del amour-propre y descubre en el fenómeno del reconocimiento recursos para promover, antes que impedir, libertad, racionalidad y virtud. El foco estará puesto en dos textos de Fichte: La destinación del académico de 1794 (BG) y un texto posterior de 1796/97: El fundamento del derecho natural (GNR). 
17 Ya en 1794, en la lecciones públicas sobre el rol del académico (Gelehrte) en la vida social, el tema del reconocimiento aparece, si bien también es claro que Fichte no había aún encontrado un rol sistemático para este concepto. ${ }^{15}$ En efecto, uno de las preguntas fundamentales de la filosofía política se dice que es: “¿cómo llegan los seres humanos a asumir y a reconocer seres racionales semejantes a ellos mismos existiendo fuera de ellos?"16 Que reconocimiento es un concepto importante ya en 1794, puede ser visto en su definición de sociedad ${ }^{17}$ como "relación de seres racionales entre sí", ${ }^{18}$ en la que los sujetos influyen unos a otros "conforme a conceptos". ${ }^{19}$ Cuando Fichte introduce aquí el tema del reconocimiento, lo hace en el contexto de definir no meramente la asociación política sino la sociedad en un sentido más general. En este texto Fichte aún cree que el destino ultimo del Estado es desaparecer una vez que haya cumplido su misión como medio para producir "una sociedad perfecta". ${ }^{20}$ Pero la desaparición del Estado no implica la desaparición de la sociedad. Por el contrario, sociedad y relaciones de reconocimiento que son constitutivas en ella se dice que pertenecen a las condiciones permanentes dentro de las cuales los seres humanos realizan su propia naturaleza en cuanto seres racionales libres y morales.

18 En la sociedad racional en la que Fichte está pensando, los individuos influyen unos a otros conforme a conceptos antes que por medio de la fuerza bruta. Esto sucede en la medida en que "reconocen" la mera razón pura ("la ley moral") ${ }^{21}$ como su "juez supremo". ${ }^{22}$ El pensamiento que se haya aquí a la base es todavía kantiano: reconocer a la razón como la autoridad suprema de uno es al mismo tiempo reconocer a los semejantes como miembros de la sociedad -tratarlos como seres con una dignidad superior a la de las meras cosas. Y esto es así debido a que la ley de la razón nos exige restringir nuestras acciones de acuerdo con la idea según la cual los otros también son fines en sí mismos.

19 La idea aquí no es meramente que la razón nos exige respetar los derechos morales de los otros cuando deliberamos acerca de lo que debemos hacer. El reconocimiento se refiere también al modo en que los miembros de una sociedad entran en relación e influyen los unos a los otros en su interacción: ellos coordinan sus acciones no por medio de relaciones jerárquicas de poder, sino "de acuerdo a conceptos", esto es, por medio del intercambio de razones. Cuando te doy razones sobre por qué deberías cooperar conmigo, y en qué términos deberías hacerlo, estoy reconociendo implícitamente tu estatus como sujeto racional -en cuanto alguien capaz de comprender y ser movido por razones- en oposición a un ser carente de racionalidad. Esta razón nos permitiría explotar, amenazar o coaccionar a los otros en la persecución de nuestros propios fines. Más aún, la igualdad de estatus está edificada en el reconocimiento de que sujetos racionales acuerdan entre ellos al reconocer a la razón como su autoridad suprema. Cuando te trato como un fin en sí mismo con tus propios fines que merecen mi respeto, o cuando te doy razones para que cooperes conmigo, estoy implícitamente considerándonos a ambos como sujetos, de igual modo, a la autoridad de la razón.

20 En este escrito temprano Fichte confunde dos preguntas distintas las cuales deberían ser respondidas por una teoría de reconocimiento. Ambas preguntas están presentes en la pregunta fundamental de la filosofía política que he citado anteriormente, a saber: “ ¿cómo es que los seres humanos llegan a asumir y a reconocer otros seres racionales semejantes a ellos?" La primera de estas preguntas es genética y concierne a cómo los individuos llegan a creer (o saber) que existen otros seres racionales; ${ }^{23}$ la segunda es 
constitutiva en el hecho de que pregunta qué significa para los seres racionales reconocerse recíprocamente, o en qué consisten las relaciones de reconocimiento entre seres racionales.

21 Tal como hemos visto, la segunda pregunta se contesta con la idea kantiana de que los seres racionales son aquellos que tienen a la razón por la máxima autoridad. No queda, sin embargo, totalmente claro de qué modo Fichte en este escrito temprano responde la primera pregunta. Por un lado, toma la idea de que en la naturaleza humana hay un "impulso básico" hacia la sociabilidad ${ }^{24}$ que nos impele "a asumir la existencia de seres racionales semejantes a nosotros". ${ }^{25}$ Por el otro, Fichte también se vale de la idea de que llego a creer que los otros también son racionales cuando veo que responden al modo en que los trato conforme la ley moral tratándome a mí también conforme a la misma ley de la razón. Uno podría decir que el reconocimiento que ellos hacen de mí -el hecho de que me traten como a un ser racional teniendo fines y mereciendo su respeto moralsirve de evidencia de que ellos también son seres racionales. Sin embargo, esto no responde en realidad la pregunta genética, la cual debe explicar cómo es que llego a la idea en primer lugar de que ellos pertenecen a la categoría "seres humanos", de la cual parece depender mi trato inicial con ellos en cuanto seres racionales. Voy a retornar a este asunto cuando discuta el GNR, pues allí Fichte invoca la idea de reconocimiento al responder la pregunta genética. Por el momento basta con notar cómo la pregunta genética aparece formulada en $B G$ : la pregunta no es cómo es que llego a considerarme a mí mismo un ser racional, sino antes bien cómo es que, siendo ya consciente de mí mismo en cuanto ser racional, aplico el mismo concepto a otros seres fuera de mí. En otras palabras, mi autoconsciencia está presupuesta y el único enigma en este texto temprano es cómo es que llego a creer que otros seres comparten mi naturaleza en cuanto ser racional.

22 Antes de que pasemos al GNR quisiera remarcar otro aspecto de este escrito temprano que es relevante para el ideal romántico de la reconciliación de los fines que tenemos como seres naturales (felicidad, supervivencia y el desarrollo de nuestras facultades) con aquellos de la razón (virtud y libertad). Como ya he indicado, Fichte dice claramente en este texto temprano que la clave para realizar la reconciliación es la cultura. Fichte define la cultura como teniendo dos partes: el cultivo de nuestros sentimientos e inclinaciones y la "modificación de cosas fuera de nosotros [...] conforme a nuestros conceptos." ${ }^{26} \mathrm{Al}$ mismo tiempo, sin embargo, la solución que ofrece Fichte al conflicto entre fines espirituales y naturales se asienta en última instancia en la naturaleza antes que en la cultura, si entendemos cultura como Rousseau lo hace, es decir, como el dominio de lo "artificial", de los quehaceres humanos libres en el sentido de que no están determinados por la naturaleza. Desde una perspectiva Rousseauniana el problema que hay con la posición de Fichte en el escrito temprano es que Fichte hace la reconciliación entre fines espirituales y naturales demasiado fácil al colocar una variedad de impulsos innatos, como por ejemplo sociabilidad, que naturalmente nos conducen a descubrir precisamente aquello que necesitamos para devenir racionales y libres. El impulso de sociabilidad, por ejemplo, se dice que comprende impulsos para educar a otros y para ser educados por ellos, y la consecuencia de tales impulsos es que la naturaleza nos conduce, más o menos directamente a establecer las relaciones de reconocimiento que son constitutivas de una sociedad racional. El hecho de que Fichte afirme precisamente lo que Rousseau había negado con tanto esfuerzo -sociabilidad natural- muestra que en este texto temprano Fichte subestima en gran medida el conflicto entre fines naturales 
y espirituales tal como Rousseau lo ha entendido. Fichte sostiene que la cultura puede reconciliar nuestros fines naturales y espirituales, pero el desarrollo al que apela es uno que está prescrito para nosotros desde un comienzo por los impulsos que la naturaleza nos ha dado.

genética. Ya no pregunta cómo es que el sujeto racional llega a tener noticia de la existencia de otros seres racionales, sino antes bien cómo un sujeto llega a su autoconsciencia en primer lugar. En 1796 Fichte ha llegado a entender que la autoconsciencia que en su escrito más temprano había meramente presupuesto, no debe ser considerada como algo dado inmediatamente, sino antes bien como una consumación, y ahora ve que esta consumación tiene precondiciones intersubjetivas: que los sujetos llegan a ser conscientes de sí mismos en cuanto racionales o libres, sólo a través de relaciones de cierto tipo con otros sujetos. En este escrito Fichte está claramente pensando la cuestión genética como una pregunta acerca del desarrollo humano y su respuesta lleva a la afirmación de la necesidad de la Erziehung, o educación, para sujetos humanos (el aspecto de la cultura que Rousseau examina en su Emile).

26 La relación específica intersubjetiva de la que aquí se trata es la Aufforderung, o exhortación. La idea es que si un sujeto es consciente de sí en cuanto fuente de actuar libre (efectividad de acuerdo a conceptos), tiene primero que encontrar este su actuar 
como un objeto de experiencia en el mundo exterior. El sujeto tiene que descubrir su libertad a través de una "intuición" externa de esta libertad que debe haber sucedido antes de haber actuado libremente (dado que actuar libremente presupone que uno ya se considera a sí mismo como libre). El GNR sostiene que el único modo para que esto ocurra es que la evidencia externa del actuar de uno sea proporcionada por otro sujeto libre bajo la forma de una Aufforderung. La Aufforderung es entonces emitida por un sujeto que ya ha sido formado a otro sujeto que aún no está formado. La Aufforderung es un llamado a actuar, un llamado a hacer realidad la actividad libre, el cual cobra la forma de un imperativo (si bien no un imperativo moral) que proclama: ¡tú debes ejercer tu actividad! Por medio de esta llamada quien ha sido exhortado aprehende la idea de su actividad libre, pero no como algo que ya existe sino como algo que debe ser realizado en el futuro. ${ }^{29}$ Más importante aún es que la exhortación en su llamado a actuar se dirige a un sujeto no educado, pero no porque se lo exija moralmente, sino simplemente porque actuar libremente es (o debería ser) un fin en sí mismo, un Endzweck, para todo ser con esta capacidad.

Dado que la Aufforderung transmite al sujeto exhortado un sentido del valor de su propio actuar, es una especie de reconocimiento, si bien se da entre dos sujetos que están situados en una relación de desigualdad (una condición inherente a Erziehung). Se trata de una forma de reconocimiento no sólo porque el educador tiene a quien recibe su mensaje por un sujeto con el potencial para actuar libremente, sino también porque al exhortarlo a actuar, quien lo hace tiene que restringir su propia libertad en relación con aquél a quien va dirigido el mensaje. La intuición externa que posee el sujeto exhortado de su propia libertad tiene que ser de tal manera que el otro sujeto le da libertad para decidir cómo actuar y esto implica por parte de quien exhorta saber dominarse para no interferir en el sujeto exhortado a actuar libremente. ${ }^{30}$ Uno podría llegar a decir que la experiencia inicial que tiene el sujeto de su libertad es una forma de Erziehung que es un prototipo de la relación intersubjetiva entre sujetos completamente formados en relaciones de derecho dentro de un orden político justo.

Esto nos lleva a la cuestión constitutiva que en el GNR se ha vuelto una pregunta acerca de cómo está conformada "una comunidad de seres libres", o cómo debe ser ordenada la interacción entre seres racionales en una sociedad justa. ${ }^{31}$ Aquí también la respuesta de Fichte apela al reconocimiento, si bien en este caso reconocimiento no es una Aufforderung de un sujeto a otro, sino una relación de derechos establecidos y fortalecidos dentro de un orden político justo entre sujetos en situación de igualdad. (En $B G$, recordemos, se decía que los sujetos en sociedad interactúan o se reconocen recíprocamente "conforme a conceptos", pero estos conceptos derivaban de la ley moral y quedaban como "meros" conceptos sin realidad social o institucional.) Los derechos defendidos en el GNR son definidos por principios que restringen las acciones de todos, de modo tal que constituyan "una esfera de libertad exclusiva y externa" 32 para cada individuo, dentro de la cual pueda ser realizado el obrar libre. Estos derechos valen como formas de reconocimiento puesto que están asentados en el principio: "todos deben [...] ser capaces de ser una persona, un [sujeto formalmente] libre" ${ }^{33}$ y en consecuencia se traducen en el requerimiento de que todos los individuos, en sus acciones exteriores, traten a todos los demás de una manera coherente con el estatus que ellos ostentan en cuanto actores formalmente libres.

29 Si bien los derechos individuales también juegan un rol particular en la visión de Rousseau acerca de la república legitima, Fichte es el primero en comprender 
explícitamente la relación de derecho entre ciudadanos en una sociedad justa en términos de reconocimiento. Es más, el hecho de que Fichte trate los derechos políticos como el paradigma principal para el reconocimiento intersubjetivo lo conduce a enfatizar un aspecto del reconocimiento que fue entendido apenas de un modo oscuro por Rousseau y que jugará un rol importante para Hegel: participar en relaciones de reconocimiento recíproco es al mismo tiempo subordinarse uno mismo $-\mathfrak{u}$ obligarse uno mismo- a una ley que es aplicada y que sujeta a todos aquellos implicados en estas relaciones. ${ }^{34}$ Por lo tanto, dentro de una corriente de pensamiento que tiene sus raíces en la idea Rousseauniana de un acuerdo entre voluntades como fuente de derecho dentro de la sociedad (en cuanto opuesto al derecho en la naturaleza) Fichte deviene el primer filósofo que localiza la fuente de la normatividad intersubjetiva en el acto de reconocimiento entre seres racionales, esto es, en reconocimiento equitativo y recíproco del valor de unos y otros en cuanto seres libres, expresado en el compromiso de cada uno de cumplir con las leyes que hacen que la realización de la libertad en el mundo exterior sea posible para todos.

Los derechos políticos que Fichte deduce de la idea de la actividad formalmente libre se dividen en dos clases: aquellos que conciernen a la inviolabilidad del cuerpo (entendido como la encarnación de la voluntad humana) y aquellos que garantizan a los individuos una esfera de "libre [acción] en el [...] mundo sensible". ${ }^{35}$ Estos últimos incluyen los derechos de auto-preservación y de la propiedad privada. Estas ideas son en general conocidas, pero tres aspectos de la doctrina de los derechos de Fichte son novedosas y dignas de atención. El primero es que Fichte ubica parte del valor de los derechos políticos en los modos en los que éstos ayudan a concretar no sólo la libertad sino también la individualidad o, como Hegel diría, la particularidad de los individuos. Por medio de crear una esfera exclusiva de acción libre para cada uno de sus miembros, un sistema de derechos políticos permite a éstos ejercer su libertad formal en cuanto actores individuales que, al obrar en sus deseos e intereses particulares, se constituyen a sí mismos como individuos particulares. En un sistema de derechos los individuos se reconocen recíprocamente como seres universales abstractos (en cuanto sujetos formalmente libres), pero este reconocimiento tiene el efecto de individualizar los seres reconocidos como tales, permitiéndoles distinguirse de sus semejantes y cobrar consciencia de sí mismos en cuanto individuos concretos.

31 Este punto conduce a la segunda innovación que presenta la comprensión fichteana de los derechos: vivir en un orden político que proteja un conjunto de derechos para todos los individuos no sólo asegura su libertad (para hacer con sus cuerpos y sus propiedades lo que decidan), también promueve un tipo de autoconsciencia, a saber, una consciencia de uno mismo en cuanto actor libre y en cuanto individuo inconfundible, lo cual incluye afirmarse o valorarse a uno mismo bajo estas descripciones. Este rol de los derechos -su contribución a formar identidades prácticas para los individuos- se sigue directamente del carácter que poseen en cuanto formas de reconocimiento y una de sus implicaciones consiste en que el ámbito político juega un rol formativo en la constitución de las concepciones que los miembros de una sociedad tienen sobre sí mismos. Los orígenes de este pensamiento pueden remontarse también a Rousseau, para quien el reconocimiento por parte de los otros es una condición necesaria para las formas saludables de auto-afirmación (incluso si las formas incorrectas de relaciones de reconocimiento pueden distorsionar el sentido que tenemos nosotros de lo que debe ser el sí-mismo). Pero Fichte, más que Rousseau, enfatiza que el reconocimiento tiene que consistir en algo más que en una opinión del valor de otro sujeto. En efecto, para Fichte 
el reconocimiento exige que los otros traten en realidad al sujeto reconocido como lo que es, que su estatus en cuanto un ser valorable sea demostrado en el mundo externo a través de la acción de estos otros.

Este es el mismo pensamiento que vimos en la doctrina de Fichte acerca de la Aufforderung: la consciencia de sí mismo de un sujeto según una cierta descripción depende de encontrar una confirmación externa y "objetiva" de esa concepción de sí mismo, la cual es lograda sólo a través de actitudes de reconocimiento ${ }^{36}$ ejercidas por otros sujetos. La diferencia aquí es la siguiente: mientras la Aufforderung es emitida por sujetos ya formados y dirigida a otros sujetos que aún no son conscientes de su libertad, los derechos políticos refuerzan las concepciones que tienen de sí mismos los sujetos adultos, al sustentar sus identidades en cuanto seres cuya libertad (e individualidad) tienen un valor ya reconocido.

La innovación final de la concepción fichteana de los derechos es la valoración positiva que le adscribe a la posesión de la propiedad, y este aspecto de su visión, también apropiada por Hegel, nos ayuda a ver cómo Fichte, a pesar de su ceguera ante los tantos peligros de la pasión por ser reconocidos de los que Rousseau era consciente, descubre un modo en el que un elemento de la cultura -la institución (hecha por el hombre) de la propiedad privada- tiene el potencial de llevar los fines naturales y espirituales a una relación de armonía. Poner el foco en el rol de los derechos políticos en hacer realidad la libertad formal y la individualidad le otorga a Fichte una razón más fuerte para defender la propiedad privada que otra que se asiente en el valor de la autopreservación o en el rol que juega la propiedad en promover la autonomía moral (Puesto que ni auto-preservación ni la autonomía moral exigen propiedad privada ${ }^{37}$ ). Si entendemos propiedad privada como Fichte lo hace, esto es, como parte del mundo sensible que está "subordinada a los fines [de uno]" y "apropiada exclusivamente en cuanto esfera de interacción [de uno]" con el mundo, ${ }^{38}$ entonces no resulta difícil ver por qué la propiedad es un derecho necesario. Si la libertad formal es completamente realizada sólo en la acción en el mundo, entonces, la sujeción (de una parte) del mundo externo a los fines particulares es necesaria para que los sujetos hagan realidad su libertad real. Es más, si un sujeto ha de constituirse a sí mismo como un individuo, cualitativamente distinto de otros seres racionales, la parte del mundo sujeta a sus fines tiene que ser exclusivamente suya, una esfera en la cual otros individuos tienen prohibido por derecho llevar a cabo sus propósitos.

Según esta descripción ser dueño de una propiedad privada y disponer de la misma según uno lo desee es una parte importante de cómo los miembros de una sociedad justa se constituyen a sí mismos y realizan su naturaleza en cuanto individuos libres que son al mismo tiempo iguales y diferentes de todos los demás, y el reconocimiento mutuo entre dueños de propiedades formalmente iguales juega un rol esencial en todo esto. Sin embargo, libertad y reconocimiento están en juego no sólo en el hecho de disponer de la propiedad de uno sin que los demás interfieran; ambos conceptos también están en juego, incluso de un modo más claro, cuando dos dueños de propiedades se encuentran para intercambiar sus pertenencias. Pues el intercambio, mediado por un contrato que vincula legalmente a las partes, es un encuentro de sujeto a sujeto en el cual la verdad que subyace sobre la propiedad -eso que "toda propiedad está basada en reconocimiento mutuo" ${ }^{39}$ - se vuelve explícita para todos y legalmente impuesta por el Estado. 
de explicar la conexión entre propiedad y libertad Fichte introduce en su descripción el rol posterior que la propiedad juega en las vidas de la mayoría de los seres humanos, a saber, en cuanto medio para satisfacer las necesidades naturales, especialmente aquellas que están ligadas con la supervivencia. Mientras la propiedad privada no puede ser justificada desde la sola perspectiva de las necesidades naturales -la propiedad común también serviría para satisfacer nuestras necesidades naturales-, una vez que la propiedad privada está establecida para realizar la libertad y la individualidad, no puede ayudar de otro modo sino jugando un rol en el modo en que seres libres se relacionan con el mundo natural en cuanto creaturas con necesidades materiales. Poseer y disponer de propiedad, podrían decir Ustedes, es una práctica humana -una parte de la cultura- dentro de la cual tanto fines naturales como espirituales pueden ser armonizados y esto es un lugar en el que se vuelve visible aquello que he estado llamando el proyecto romántico de Fichte. Más aún, si el intercambio es una manifestación aún más vívida de las relaciones de reconocimiento que abarcan la libertad, entonces el comercio -un fenómeno que parece jugar sólo un rol negativo en el Segundo discurso de Rousseau- también tiene el potencial de reconciliar fines espirituales y naturales dentro de una sola actividad.

Al igual que Rousseau, Fichte es consciente de las consecuencias destructivas que puede tener el comercio irrestricto y la inequidad económica para una sociedad, pero él muestra también que si el comercio y la inequidad son restringidos de modo apropiado ellos tienen el potencial de ser lugar para la libertad y autorrealización de los individuos humanos. Esta idea representa un atajo a través del cual, parafraseando a Kant, la cultura puede desplegar las capacidades de la humanidad de tal modo que nuestra destinación en cuanto especie espiritual ya no entre en conflicto con nuestro carácter como especies naturales.

\section{NOTAS}

1. Fichte dedica una lección entera al Segundo discurso en su Algunas lecciones sobre la destinación del académico de 1794 (BG, GA I/3, 59-68), precisamente en el contexto de discutir estas cuestiones fundamentales.

2. Kant, I., "Muthmaßlicher Anfang der Menschengeschichte" (1786), AA 08, 116.

3. Como evidencia véase que Fichte en su quinta lección sobre el destino del académico no menciona problema alguno referido al "amour-propre" rousseauniano.

4. BG, GA I/3, 31. Véase también BG GA I/3, 41 y 45.

5. Por ejemplo el "impulso hacia la sociabilidad" (gesellschaftliche Trieb) en cuanto "impulso fundamental en el hombre" (ein Grundtrieb des Menschen) consistente en buscar fuera de sí mismo otros seres racionales semejantes a él. (BG, GA I/3, 37) Este impulso incluye el "impulso hacia la comunicación" y el "impulso hacia la recepción" de aquello que nuestros semejantes quieren comunicarnos. (BG, GA I/3, 44)

6. Fichte presupone la existencia de una "trayectoria del género humano ya prefijada a priori" (BG, GA I/3, 37) en cuya realización actúan impulsos para lograr una coordinación de las acciones

Revista de Estud(i)os sobre Fichte, 7 | 2013 
de los individuos. Véase también el rol positivo de las necesidades naturales en el perfeccionamiento de la sociedad. (BG, GA I/3, 37-38) De allí que Fichte luego pueda decir: "Ahora se puede [...] calcular la marcha del género humano [e] [...] indicar los pasos específicos [...] a transitar". (BG, GA I/3, 53) Esta historia a priori no garantiza el progreso sino la posesión de ciertos impulsos tendiente a llevarnos en la dirección correcta.

7. Cuando Fichte retoma la pregunta del Segundo discurso, a saber, “¿De dónde ha surgido la inequidad [moral] entre los hombres [sc. la diferencia entre los estamentos sociales]?” (BG, GA I/ $3,42)$, en su respuesta no apela en absoluto a la noción de amour-propre, sino que remite sencillamente la inequidad en la sociedad a una combinación entre inequidad física y libertad individual de elección.

8. En este respecto Fichte podría haber aprendido más de Rousseau de lo que realmente aprendió. Sus esperanzas demasiado optimistas acerca del destino de la historia de la humanidad son una consecuencia de esto.

9. En este sentido vale decir que la visión hegeliana sobre el potencial positivo del impulso de reconocimiento depende en parte del tratamiento que dio Fichte a la misma idea (si bien, según veo yo, Hegel comprende mejor que Fichte las razones que llevaron a Rousseau a considerar este impulso como una amenaza constante para el bienestar del hombre).

10. "Servitude" y "asservir" son sinónimos de "dominación" “("domination")y "dominar" (“dominer"), cf. Rousseau, J.-J., CEuvres Complètes, Gagnebin, B. \& Raymond, M. (eds.), Paris: Gallimard, Bibliothèque de la Pléiade, 1959-69, vol. 3, 161-162.

11. Rousseau, J.-J., Euvres Complètes, 3, 171.

12. Smith, A., The Wealth of Nations, ed.by E. Cannan, New York: Modern Library, 2000, 75-76.

13. Los aspectos de la dependencia humana originados en el amour propre, aun cuando no impliquen posesión de bien material alguno, también juegan un rol significativo en el hecho de hacernos dependientes de los demás $\mathrm{y}$, de este modo, ponen en riesgo nuestra libertad. Si, por ejemplo, quiero que tengas una buena opinión sobre mi, puede suceder que me vea tentado a actuar de un modo que no yo sino tú deseas, y caer en esta tentación significa ya haber obedecido tu voluntad antes que la mía.

14. En su Segundo discurso, se entiende.

15. Fichte no utiliza el término 'Anerkennung' en estas lecciones, pero emplea el verbo 'anerkennen 'en más de una ocasión.

16. BG, GA I/3, 34 .

17. A diferencia del GNR, en BG Fichte utiliza indistintamente los términos 'Gesellschaft' and ' Gemeinschaft' (GA I/3, 37).

18. BG, GA I/3, 34 .

19. BG, GA I/3, 37 .

20. Ibíd.

21. BG, GA I/3, 38 .

22. BG, GA I/3, 37 .

23. Ciertamente se podría afirmar que Fichte aquí no está formulando la pregunta genética, sino antes bien la pregunta acerca de ¿qué evidencia tenemos para creer que existen seres racionales fuera de nosotros? Tal vez ésta sea la mejor interpretación, sin embargo las referencias a un Grundtrieb dan a entender que Fichte está haciendo la pregunta genética. $O$ dicho incluso de mejor modo: tal vez Fichte está confundido aquí. En todo caso, él sí hace la pregunta genética en su GNR.

24. BG, GA I/3, 37.

25. Ibíd.

26. BG, GA I/3, 31 .

27. Esto es lo que Fichte llama "libertad (formal)". (GNR, GA I/3, 351 y 404) Esta libertad consiste en la capacidad para elegir libremente. La persona elige entre un conjunto de acciones posible y 
elige libremente porque su elección es llevada a cabo de acuerdo con una concepción de un fin la cual supone espontaneidad. (GNR GA I/3, 349-351)

28. GNR, GA I/3, 359.

29. GNR, GA I/3, 342-343.

30. Ibíd.

31. En el punto II del $\S 7$ del GNR (GA I/3, 385) Fichte pone en claro que a diferencia de lo que ha dicho antes, no considera las relaciones de derecho como condiciones de la autoconsciencia, sino como bases para la regulación de relaciones entre seres ya autoconscientes. En este texto Fichte tiende a utilizar Gemeinschaft en lugar de Gesellschaft, mientras que en BG, como ya he dicho, utiliza ambos términos indistintamente. (véase supra nota 17)

32. Véase distintas variaciones de esta frase en GNR, GA I/3, en: SW, III, pp. 353, 357 y 361.

33. GNR, GA I/3, 390.

34. En un lenguaje que recuerda la concepción de Brandom de reconocimiento Fichte dice: "me remito a una ley que vale para nosotros dos [...] Por esto me pongo en cuanto juez [...] Pero al referirme a aquella ley general contra él, lo estoy invitando a juzgar conmigo al mismo tiempo y lo exhorto a que tenga que reconocer que en este caso he procedido de modo consecuente contra él y así tenga que aprobarlo" (GNR, GA I/3, 356).

35. GNR, GA I/3, 409.

36. Actitudes prácticas, por supuesto, no meras "opiniones".

37. Resulta difícil ver en qué medida una teoría que deduce de los requerimientos de una voluntad substancialmente auto-determinada derechos naturales podría desarrollar un derecho inalienable a la propiedad privada sin el concepto de personalidad. Si bien el Sistema de la doctrina de las costumbres de 1798 (SSL) establece un deber moral positivo a adquirir propiedad, esto sucede sólo por medio del concepto de personalidad y el deber moral de realizar la libertad formal individual (SSL, GA I/5, 246, 265, §§ 22-23). Fichte mismo admite esto en su escrito de 1793 acerca de la revolución francesa, cuando llega a la conclusión de que el derecho de propiedad podría ser alienado ya sea en parte ya sea en su totalidad. (BB, GA I/1, 315)

38. GNR, GA I/3, 407 y GA I/4, 20.

39. GNR, GA I/3, 418.

\section{RESÚMENES}

In the present paper I intend to investigate Fichte's use of the idea of Anerkennung, or recognition -the end that our amour-propre leads us to seek - in his positive account of how humans can realize, all at once, virtue, freedom, and rationality. In other words, I will examine how Fichte appropriates Rousseau's complex theory of amour-propre and human recognition. My claim is that even though Fichte underestimates the dangers to human well-being posed by our drive to be recognized by others, he can be credited with discovering in that same human need a potential for avoiding those dangers that Rousseau was only dimly aware of.

\section{ÍNDICE}

Keywords: Fichte, Rousseau, romanticism, recognition, amour-propre 
AUTORES

FREDERICK NEUHOUSER

Barnard College 